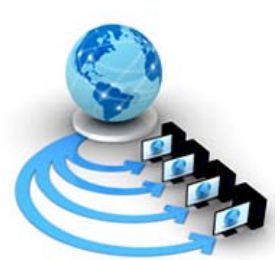

\title{
SECURITY BASED VEHICULAR NETWORKS USING CONGESTION CONTROL
} ALGORITHM

\author{
K.Ravikumar \\ Dept: Computer Science \\ Tamil University, \\ Thanjavur-613010, India,
}

\author{
T.Vishvaroobi \\ Dept: Computer Science \\ Tamil University, \\ Thanjavur-613010, India,
}

\begin{abstract}
Supportive inter vehicular applications frequently on replace of transmit single-hop messages with vehicles on a particular power channel, which present in depth sequence on the vehicle location velocity, heading, quickening, and other numbers of importance In this thesis, they apply the network utility maximization (NUM) methodology with a different approach: First, since standards allow the use of different transmit power levels, they assume that vehicles an use a set of transmit power levels and select a particular beaconing rate for each power in the set, transmitting at multiple power levels with different rates during the cycle. Network interfaces for vehicular networks actually allow for setting the transmit power for each individual frame. Second, the optimization variable used in the utility function is not simply the

Beaconing rate but the beaconing rate used with one power multiplied by the number of neighbors reached with that power. This new variable counts the total number of copies of a beacon that are delivered (in the absence of errors) in its neighborhood, and hence, it provides a measure of the degree of dissemination of the state of a given vehicle. Thus, they call this new variable the beacon dissemination rate (BDR). Each vehicle seeks to maximize the BDR, which can also be seen as a measure of the awareness its neighbors have of it. From this model, they derive a particular distributed algorithm, with guaranteed convergence to a fair allocation and which is remarkfilekjible since vehicles can independently and dynamically adapt the algorithm parameters to the requirements of a wide range of applications. For instance, it can be seamlessly used to implement prioritized congestion control, in the sense that vehicles with special needs are allocated higher rates to disseminate their status more frequently. In fact, the use of multiple power levels in a cycle, which is a novel approach compared with previous proposals, supports the parallel execution of multiple applications, with different quality-of-service requirements, on top of it. Replication consequences validate our approach and confirm that it provide fair rate allocation in realistic various and dynamic scenario by means of packet losses.
\end{abstract}

Keywords: Vehicular networks, beaconing rate, congestion control, transmit powers, broadcast messages.

\section{INTRODUCTION}

Vehicular networks are a novel class of wireless networks that have appeared recognitions to advances in wireless technologies and the automotive industry [4]. Vehicular networks are spontaneously formed between moving vehicles equipped with wireless interfaces that could be of homogeneous or heterogeneous technologies [9]. These networks, also known as VANETs, are considered as one of the ad hoc network real-life application enabling communications between nearby vehicles as well as between vehicles and nearby fixed equipment, usually described as roadside equipment [7][2]. Vehicles can be either private, belonging to individuals or private companies, or public transportation means (e.g., buses and public service vehicles such as police cars) [5]. Fixed equipment can belong to the government or private network operators or service providers [1]. Actually, vehicular networks are promising in allowing diverse communication services to drivers and passengers [6].

Internet can be reflect a row of packets, where transmitting nodes are regularly adding packets and a few of them are remove packets from the queue [10]. So, regard as a situation where too many packets are present on this queue. This reduce the performance, and such a condition is term like Congestion. The important basis of congestion is added number of packets into the network[3].therefore, the purpose of congestion control can be concise to maintain the number of packets in the network.

\section{A) A Network of Queues}

- $\quad$ At each node, there is a queue of packets for each outgoing channel

- If the packet arrival rate exceeds the packet transmission rate, the queue size grows without bound

- $\quad$ When the line for which packets are queued becomes more than $80 \%$ utilized, the queue length grows alarmingly

\section{PROBLEM DEFINITION}

Intervehicle communications based on wireless technologies have paved the way for innovative applications in traffic safety, driver assistance, traffic control, and other advanced services that will make up future intelligent transportation systems.

\section{EXISTING SYSTEM}

Fairness must be guaranteed as a safety requirement since beacons are used to provide vehicles with an accurate estimate of the state of their neighbors or mutual awareness. The parity of plan imply that no vehicle should be allocated at random less resources under the control required by the offered capacity. but still starting from the previous rule, a few ideas of fairness can be dened, and there is a exchange between fairness andfieifency. whereas, less ability is damaging to safety, in general, the high beaconing rate, high quality of the state information. 
Thus, an idea of fairness involve not only wasting resources but also has a damaging rudeness on the safety of the user

\section{A) Demerits}

- $\quad$ Less efficient use of the shared resource.

- Less security

- More time to taken to transfer data.

\section{PROPOSED SYSTEM}

The propose the network utility maximization (NUM) methodology with a different approach: First, since standards allow the use of different transmit power levels, they assume that vehicles an use a set of transmit power levels and select a particular beaconing rate for each power in the set, transmitting at multiple power levels with different rates during the cycle. Network interfaces for vehicular networks actually allow for setting the transmit power for each individual frame. Second, the optimization variable used in the utility function is not simply the beaconing rate but the beaconing rate used with one power multiplied by the number of neighbors reached with that power. This new variable counts the total number of copies of a beacon that are delivered (in the absence of errors) in its neighborhood, and hence, it provides a measure of the degree of dissemination of the state of a given vehicle. Thus, they call this new variable the beacon dissemination rate (BDR).From this model, they derive a particular distributed algorithm, with guaranteed convergence to a fair allocation and which is remarkathexible since vehicles can independently and dynamically adapt the algorithm parameters to the requirements of a wide range of applications.

\section{A) Merits}

- $\quad$ Allow the use of different transmit power levels.

- Allow for setting the transmit power for each individual frame.

- $\quad$ Provide time flexible for data allocation.

\section{EXPERIMENTAL RESULT}

\section{A) High Performance}

In these experiments High Performance. DFB gives a result with throughput of $30 \%$. VN gives a result with throughput of $38 \%$. CAC gives a result with throughput of $40 \%$. GCC gives a result with throughput of $60 \%$.

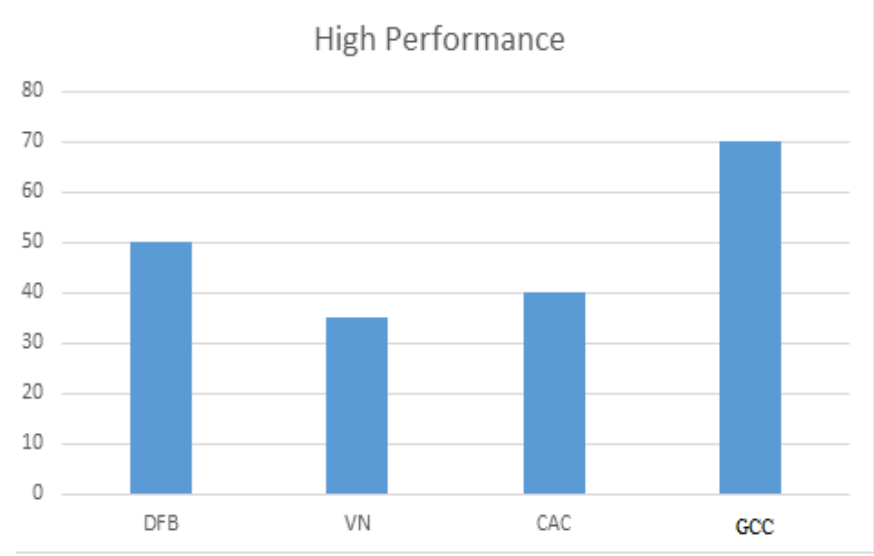

\section{B) Cost}

In these experiments High Performance. DFB gives a result with throughput of $75 \%$. VN gives a result with throughput of $60 \%$. CAC gives a result with throughput of $50 \%$. GCC gives a result with throughput of $30 \%$.

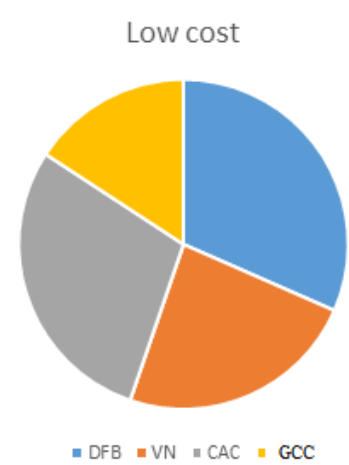

\section{C) Maximum Throughput}

In these experiments High Performance. DFB gives a result with throughput of $40 \%$. VN gives a result with throughput of $35 \%$. CAC gives a result with throughput of $20 \%$. GCC gives a result with throughput of $60 \%$.

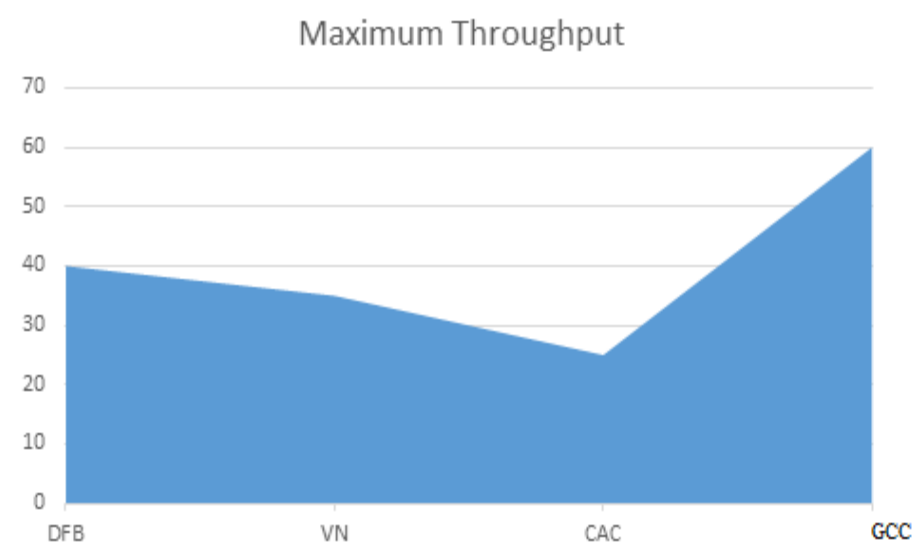

\section{CONCLUSION}

The network utility maximization (NUM) methodology with a different approach: First, since standards allowed the use of different transmit power levels. Second, the optimized variable used in the utility function is not simply the beaconing rate but the beaconing rate used with one power multiplied by the number of neighbors reached with that power. This new variable counts the total number of copies of a beacon that are delivered (in the absence of errors) in its neighborhood, and hence, it provided a measure of the degree of dissemination of the state of a given vehicle.

\section{FUTURE WORK}

The future work persist, they will improve the performance of fair allocation. to further explore variations of the discussed problem in the context of vehicular networks, particularly a comparative application and evaluation of alternative fairness notions and the 
introduction of heterogeneous utility functions and constraints in the problem.

\section{REFERENCES}

[1] Esteban Egea-Lopez and Pablo Pavon-Marino, "Distributed and Fair Beaconing Rate Adaptation for Congestion Control in Vehicular Network”, 2016.

[2] Miguel Sepulcre, Javier Gozalvez, “Adaptive Beaconing for Congestion and Awareness Control in Vehicular Networks", July 2014.

[3] Esteban Egea-Lopez, Juan J. Alcaraz, Javier Vales-Alonso, "Statistical Beaconing Congestion Control for Vehicular Network”, 2013.

[4] Yaser P. Fallah, Ching-Ling Huang, Raja Sengupta, "Analysis of Information Dissemination in Vehicular AdHoc Networks With application to Cooperative Vehicle Safety Systems”, 2011.
[5] Georgios Karagiannis, Onur Altintas, Eylem Ekici, "Vehicular networking: A survey and tutorial on requirements, architectures, challenges, standards and solutions”, 2011.

[6] Miguel Sepulcre, Jens Mittag, "Congestion and Awareness Control in Cooperative Vehicular Systems”, 2011.

[7] John B. Kenney, Gaurav Bansal, "LIMERIC: A Linear Message Rate Control Algorithm for Vehicular DSRC Systems”, 2011.

[8] T. Tielert, D. Jiang, Q. Chen, "Design Methodology and Evaluation of Rate Adaptation Based Congestion Control for Vehicle Safety Communications”, 2011.

[9] Ching-Ling Huang, Yaser P. Fallah, "Adaptive Intervehicle Communication Control for Cooperative Safety Systems”, 2010.

[10] Tian Lan , David Kao, “An Axiomatic Theory of Fairness in Network Resource Allocation”, 2010. 